$24.6 \pm 7.8 \%, p<0.0001)$, and a trend to decreased visceral fat weight $(0.9 \pm 0.9$ vs. $0.5 \pm 0.5 \mathrm{~kg}, \mathrm{p}=0.0670$ ). Compared to HC, SSc patients demonstrated significantly decreased lean body mass assessed by both iDXA (LBM: $46.6 \pm 7.5$ vs. $40.9 \pm 6.8 \mathrm{~kg}$, $\mathrm{p}=0.0003$ ) and BIA (LBM: $53.2 \pm 8.7$ vs. $47.7 \pm 7.0 \mathrm{~kg}, \mathrm{p}=0.0017$ ), and increased $\mathrm{ECM} / \mathrm{BCM}$ ratio (extracellular mass/body cell mass: $1.03 \pm 0.1$ vs. $1.29 \pm 0.4$, $\mathrm{p}<0.0001$ ), which reflects worse muscle predispositions for physical exercise, aerobic fitness/performance, and usually increases with deteriorating nutritional status. Compared to HC, SSc patients had significantly lower bone mineral density (BMD: $1.16 \pm 0.10$ vs. $1.05 \pm 0.11 \mathrm{~g} / \mathrm{cm}^{2}, p<0.0001$ ), and were currently able to perform less energetically demanding physical activities according to HAP score ( $84.7 \pm 6.6$ vs. $64.1 \pm 17.2, p<0.0001)$. Disease activity negatively correlated with $\mathrm{BF} \%(\mathrm{r}=-0.324, \mathrm{p}=0.014)$, and physical activity (HAP) positively correlated with $\operatorname{BMD}(r=0.276, p=0.034)$ and negatively with $\mathrm{ECM} / \mathrm{BCM}(\mathrm{r}=-0.625, \mathrm{p}<0.0001)$.

Conclusions: Compared to healthy age-/sex-matched individuals we found significant negative changes in body composition of our SSc patients, which are associated with their disease activity and physical activity, and could reflect their nutritional status, and gastrointestinal and musculoskeletal involvement.

Acknowledgements: Supported by AZV-16-33574A, GAUK-214615.

Disclosure of Interest: None declared

DOI: 10.1136/annrheumdis-2017-eular.4873

\section{SAT0359 THE COURSE OF MOUTH OPENING AND ITS RELATIONSHIP WITH DISEASE CHARACTERISTICS, GLOBAL FUNCTIONING, HEALTH-RELATED QUALITY OF LIFE AND MOUTH HANDICAP IN PATIENTS WITH SYSTEMIC SCLEROSIS}

S.J.H. Khidir ${ }^{1}$, M. Boonstra ${ }^{1}$, G.W.M. Boerrigter ${ }^{2}$, E.M. Voogt-van der Harst ${ }^{2}$, S.A. Bergstra ${ }^{1}$, M.K. Ninaber ${ }^{3}$, N. Ajmone Marsan ${ }^{4}$, T.W.J. Huizinga ${ }^{1}$, J.K. de Vries-Bouwstra ${ }^{1} .{ }^{1}$ Rheumatology; ${ }^{2}$ Physiotherapy; ${ }^{3}$ Pulmonology; ${ }^{4}$ Cardiology, Leiden University Medical Center, Leiden, Netherlands

Background: Systemic sclerosis (SSc) is a fibrotic disease which can lead to reduced maximal mouth opening (MMO). Previous cross-sectional research showed that reduced MMO in SSc correlated with higher disease severity and lower oral quality of life. Interpretation of interventions that possibly influence $\mathrm{MMO}$ is difficult, as the natural history of MMO in SSc is not well described.

Objectives: To evaluate in SSc 1) the course of MMO 2) disease characteristics predictive for decreasing $\mathrm{MMO}$ and 3) the relationship between the course of MMO and global functioning, health-related quality of life (HRQoL) and mouth handicap.

Methods: SSc patients from the Leiden Combined Care In Systemic Sclerosis (CCIS) cohort, Leiden University Medical Center were included if at least one MMO measurement was available. Annual clinical assessment includes MMO

Table 1. Multivariate mixed model to determine the association between Maximal Mouth Opening (MMO) and baseline disease characteristics in SSc patients

\begin{tabular}{|c|c|c|c|c|}
\hline & \multirow[t]{2}{*}{$\beta$} & \multirow[t]{2}{*}{$p$-value } & \multicolumn{2}{|c|}{ 95\% Confidence Interval } \\
\hline & & & Lower limit & Upper limit \\
\hline Non cutaneous SSc (3) & & & ref & \\
\hline Limited SSc (2) & -2.2 & 0.036 & -4.2 & -0.2 \\
\hline Diffuse SSc (1) & -4.1 & 0.004 & -6.9 & -1.4 \\
\hline Gastrointestinal involvement & -2.6 & 0.001 & -4.1 & -1.1 \\
\hline Pulmonary involvement & -1.8 & 0.032 & -3.4 & -0.2 \\
\hline Peripheral vasculopathy & -2.3 & 0.003 & -3.8 & -0.8 \\
\hline
\end{tabular}

Figure 1 Mean Maximal Mouth Opening (MMO), percentage of microstomia and change in MMO over time in SSc patients

Mean MMO and percentage of microstomia over time

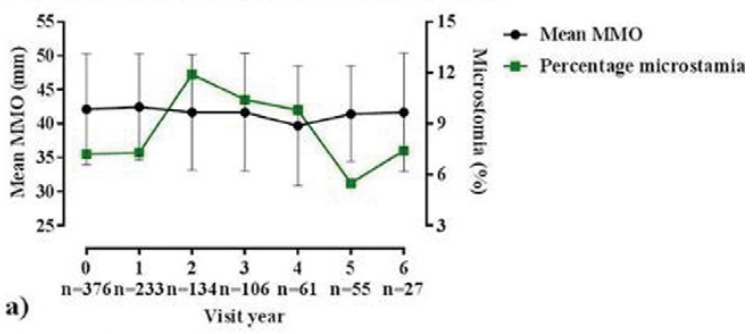

Change in MMO between consecutive visits

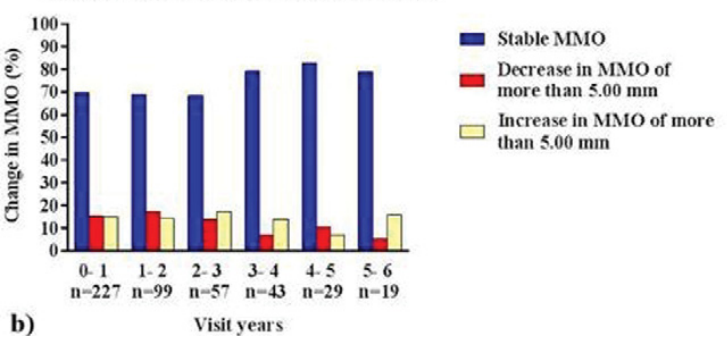

measurement, global functioning (HAQ), HRQoL (Short Form-36; SF-36), mouth handicap (Mouth Handicap in Systemic Sclerosis scale; MHISS). We assessed mean $\mathrm{MMO}$, prevalence of microstomia $(\mathrm{MMO}<30.00 \mathrm{~mm})$ and decreasing $\mathrm{MMO}$ (decline $>5.00 \mathrm{~mm} /$ year) over time. Predictors for decreasing $\mathrm{MMO}$ were assessed by a linear mixed model (LMM), including baseline clinical parameters reflecting 7 domains (peripheral vasculopathy, skin, heart, gastrointestinal, lung, renal, musculoskeletal). Additionally, MMO over time was correlated with baseline HAQ, SF-36 and MHISS in separate LMMs adjusted for gender, Body Mass Index, age and disease characteristics correlated significantly $(p<0.05)$ with the course of MMO.

Results: 382 patients were included with mean age $54 \pm 14$ years, $83 \%$ female and $25 \%$ diffuse cutaneous SSc. Mean MMO during 6 years of follow-up ranged from 39.4 to $42.5 \mathrm{~mm}$. The annual mean percentage of patients with microstomia was $9 \%$, range 6 to $12 \%$ (Figure 1a). A decrease in MMO between two consecutive annual measurements was observed in 5 to $17 \%$ of patients, mean $12 \%$ (Figure $1 b)$. More extended cutaneous involvement, peripheral vasculopathy, pulmonary and gastrointestinal involvement at baseline were predictive for decreasing MMO over time (Table 1). Baseline HAQ ( $\beta=-1.6,95 \% \mathrm{Cl}=-2.7$ to -0.6$)$, SF-36 physical component $(\beta=0.1,95 \% \mathrm{Cl}=0.0$ to 0.1$)$ and $\mathrm{MHISS}(\beta=-0.2,95 \% \mathrm{Cl}=-0.2$ to -0.1$)$ correlated with longitudinal MMO.

Conclusions: Over time, MMO is relatively stable in the majority of SSc patients. Microstomia is seen in $9 \%$ and important decrease of MMO in $12 \%$ and is associated with more severe organ involvement. Even though this concerns only a small subgroup of SSc patients, a significant association with global functioning and HRQoL was demonstrated, underlining the need for treatment strategies improving MMO.

Disclosure of Interest: None declared

DOI: 10.1136/annrheumdis-2017-eular.6245

\section{SAT0360 MYOSITIS-SPECIFIC AND MYOSITIS-ASSOCIATED AUTOANTIBODIES IN PATIENT WITH DERMATOMYOSITIS / POLYMYOSITIS; COMPARISON BETWEEN LINE BLOT AND ENZYME-IMMUNOASSAY ASSAYS}

S. Nakashima, T. Kameda, M. Izumikawa, H. Shimada, H. Ozaki, R. Wakiya, A. Kondou, N. Kadowaki, H. Dobashi. Department of Internal medicine, Division of Hematology, Rheumatology and Respiratory Medicine, Kagawa University, Kagawa, Japan

Background: MESACUPTM test (enzyme-immunoassay assays; TIF1 $\gamma$, MDA5, Jo-1, EJ, PL-7, PL-12, and KS; MBL) (MESA) is used for a diagnosis of idiopathic inflammatory myopathies (IIMs) in Japan. On the other hand, EUROLINE myositis Profile 3 (line blot; EUROIMMUN) (EURO) can analyze plural Myositis-specific autoantibodies (MSA: Mi-2, Jo-1, SRP, PL-7, PL-12, EJ, OJ) and Myositisassociated autoantibodies (MAA; Ku, PM-Scl) at the same time, which is used commercially in Western countries. However, the difference between utility of MESA and that of EURO haven't be disclosed.

Objectives: To clarify difference between utility of EURO and MESA, and extract the problem of respective examination.

Methods: We enrolled 58 patients diagnosed DM/PM in our facility. Polymyositis (PM) and dermatomyositis (DM) were diagnosed according to Bohan and Peter's criteria. ${ }^{1)}$ The MAA and MSA were analyzed using MESA and EURO. In case of MESA (+), MSA (anti-Jo1, anti-PL7, anti-PL12, anti-KS) were identified by specific ELISA. When those results were different, we analyzed by immunoprecipitation. And we analyzed the association between each autoantibody and clinical features. Results: MSA and MAA were detected in 43/58 (74\%) (anti-PL7: 12, anti-Jo1: 7 , anti-EJ: 3, anti-PL12: 1, anti-OJ: 0, anti-Ro52: 27, anti-PM-Scl75: 7, anti-Ku: 6, anti-PM-Scl100: 1) by EURO. On the other hand, MSA and MAA were detected in 30/58 (52\%) (anti-PL7: 9, anti-Jo1: 7, anti-EJ: 4, TIF1 $\gamma$ : 4, MDA5: 3, U1RNP: 3) by MESA. Five patients were MESA (-) and EURO (-). In the case of ARS positive patient, Two of EURO (-) patients was positive in MESA, respectively Jo1 and EJ. Three of MESA (-) patients was positive in EURO. Although MESA(-) and $\mathrm{EURO}(+)$ patients had plural MSA and MAA (PL7+Jo1, PL7+PL12, PL7+Ku), MSA and MAA weren't detected by immunoprecipitation in MESA(-) and EURO(+) patients. Two of patients that detected plural MSA or MAA had rapid progressive ILD.

(Association between clinical manifestations and MSA, MAA)

All patients with anti-ARS (anti-Jo-1, anti-PL-7, anti-PL-12 and anti-EJ) had ILD. In addition, anti-ARS were associated with arthritis and mechanic's hands. Anti-Mi-2 positive patients didn't have ILD. Patients detected anti-PM-Scl75, anti-PM-Scl100, anti-Ku were almost overlap syndrome. All of Anti-SRP positive patients was PM.

Conclusions: EURO is a convenient and reliable method useful for detection of MSA and MAA. It was suggested the patients whom plural antibodies were detected by EURO have unique clinical course in others.

References:

[1] Bohan, A., Peter, J.B., 1975. Polymyositis and dermatomyositis (second of two parts). N. Engl. J. Med. 292, 403-407.

Disclosure of Interest: None declared

DOI: 10.1136/annrheumdis-2017-eular.4762 